\title{
Efficient Design of Digital FIR Differentiator using $L_{1}$-Method
}

\author{
Apoorva AGGARWAL, T. K. RAWAT, Manjeet KUMAR, D. K. UPADHYAY \\ Division of Electronics and Communication Engineering, Netaji Subhas Institute of Technology, \\ Sector-3, Dwarka, New Delhi 110078, India
}

\{16.apoorva, tarundsp, manjeetchhillar\}@gmail.com, upadhyay_d@ rediffmail.com

Manuscript received October 9, 2015

\begin{abstract}
In this paper, an efficient design of FIR digital differentiator using the $L_{1}$-optimality criterion is proposed. We present a technique based on the modified Newton method to solve the design problem so that the optimal differentiator coefficients are obtained by minimizing the absolute error. The novel $L_{1}$-error function leads to a flat response at low-frequencies. Extensive simulations are carried out to validate the proposed design. The superiority of the proposed design is evident by comparing it with other conventional design techniques such as, windowing, minimax and the least-squares approach.
\end{abstract}

\section{Keywords}

FIR differentiator, $L_{1}$-method, digital filter, magnitude response

\section{Introduction}

In recent years, design of digital differentiator has emerged as an important research area in the field of signal processing. Digital differentiators are used to extract information about rapid transients by computing the time derivatives of the signal. Digital differentiators are broadly applied in the field of radar and sonar applications [1], robotics and control engineering [2], [3], communication systems [4], biomedical signal processing [5], speech and image processing [6] and seismic systems [7]. With these wide range of applications, the design of digital differentiators are extensively researched over a few decades.

The prevalent approaches to design digital differentiator are based on different interpolation and system approximation based techniques [8-15] and optimization based techniques [16-21]. The design of digital differentiator based on system approximation is a four-step process. First, define a desired frequency response of the digital differentiator. Second, select the type of system (either $(N-1)$ th order FIR or IIR). Third, develop an optimality criterion in order to approximate the ideal response. In this work, the $L_{1}$ opti- mality criterion is adopted. Lastly, establish an analytical computational method to compute the optimal differentiator.

In this paper, mathematical modelling for the design of the digital differentiator based on the type-4 FIR filter approximation using the $L_{1}$-method is proposed. The FIR filter is considered due to its inherent stability and linear phase characteristics. The $L_{1}$ approach computes the $L_{1}$-norm of the approximation function. The motivation of implementing the $L_{1}$-method for the differentiator design, is due to its ability to produce the flattest response that approaches to the ideal one. The $L_{1}$ differentiator design is collated with other design techniques, namely windowing method, minimax design and the least-squares approach. The novelty of the presented paper is the integration of procured minimum absolute magnitude error and flatness in response using the $L_{1}$ method.

The rest of the paper is organized as follows. The problem formulation of $L_{1}$ differentiators is introduced in Sec. 2. In Sec. 3, mathematical modelling of the $L_{1}$-algorithm for the design of type-4 FIR digital differentiator is described. The results obtained are pictured and analyzed in Sec. 4. Finally in Sec. 5, the proposed design is concluded with the advantageous observations.

\section{Digital Differentiator Design Prob- lem}

The frequency response of the ideal $(N-1)$ th order digital differentiator is given by

$$
\begin{aligned}
& H_{i d}(\omega)=|\omega| \mathrm{e}^{\mathrm{j} \frac{\pi}{2} \operatorname{sgn}(\omega)}, \quad-\pi \leq \omega \leq \pi \\
& H_{i d}(\omega)=\left\{\begin{array}{lr}
\omega \mathrm{e}^{\mathrm{j} \frac{\pi}{2}}, & 0 \leq \omega \leq \pi \\
-\omega \mathrm{e}^{-\mathrm{j} \frac{\pi}{2}}, & -\pi \leq \omega<0
\end{array}\right.
\end{aligned}
$$

where $\operatorname{sgn}(\omega)$ gives the Signum result of $\omega$. The ideal differentiator is approximated to the $(N-1)$ th order FIR filter with impulse response $\{h(k), 0 \leq k \leq N-1\}$ in order to design a digital differentiator. 
The frequency response of the $(N-1)$ th order FIR filter to be designed is given by

$$
H(\omega)=\sum_{k=0}^{N-1} h(k) \mathrm{e}^{-\mathrm{j} \omega k} .
$$

Since the frequency response of the digital differentiator, $H_{i d}(\omega)$, is purely imaginary and odd, the coefficients will be antisymmetric $\{h(k)=-h(N-1-k)\}$. This implies that either a type-3 or a type-4 FIR filter can be used for designing of digital differentiator. In this paper, design of type-4 FIR digital differentiator is considered. The frequency response of type-4 FIR filter is given by

$$
H(\omega)=\mathrm{e}^{\mathrm{j}\left(\frac{\pi}{2}-\omega M\right)} \sum_{k=1}^{M} d(k) \sin (\omega(k-0.5))
$$

where $M=N / 2$ and $d(k)=2 h(M-k), 1 \leq k \leq M$ are the filter coefficients. The absolute response is defined as

$$
D(\omega)=\sum_{k=1}^{M} d(k) \sin (\omega(k-0.5))
$$

With this type of filter the differentiator can be designed for the complete frequency range, since it has a zero at $\omega=0$ only. In order to obtain the digital differentiator, $H_{i d}(\omega)$, with the desired specifications, it is required to design the FIR filter by determining the optimal filter coefficients and approximating it with the ideal differentiator. An objective function in terms of the error between the ideal frequency response and the FIR filter response is developed. For this purpose, the absolute weighted error is minimized to obtain a set of optimized coefficients. The $L_{1}$ weighted error function is defined as

$$
\varepsilon=\int_{0}^{\pi} W(\omega)|E(\omega)| \mathrm{d} \omega
$$

where $E(\omega)=H_{i d}(\omega)-H(\omega)$ and $W(\omega)$ is the non-negative weighted function. For type-4 differentiator, the fitness function is written as

$$
\varepsilon=\int_{0}^{\pi} W(\omega)\left|H_{i d}(\omega)-\sum_{k=1}^{M} d(k) \sin (\omega(k-0.5))\right| \mathrm{d} \omega .
$$

In this paper, the problem is to compute the filter coefficients, $d(k)$ on the minimization of the fitness function defined in (6).

\section{Mathematical Framework for the Design of $L_{1}$ Differentiator}

The $L_{1}$ algorithm developed for the design of FIR linear phase filters [22-25], is successful in optimizing the type1 FIR filter coefficients and the designed filter yields a smallest overshoot around the discontinuity along with flattest response in the passband as compared to least-square, minimax

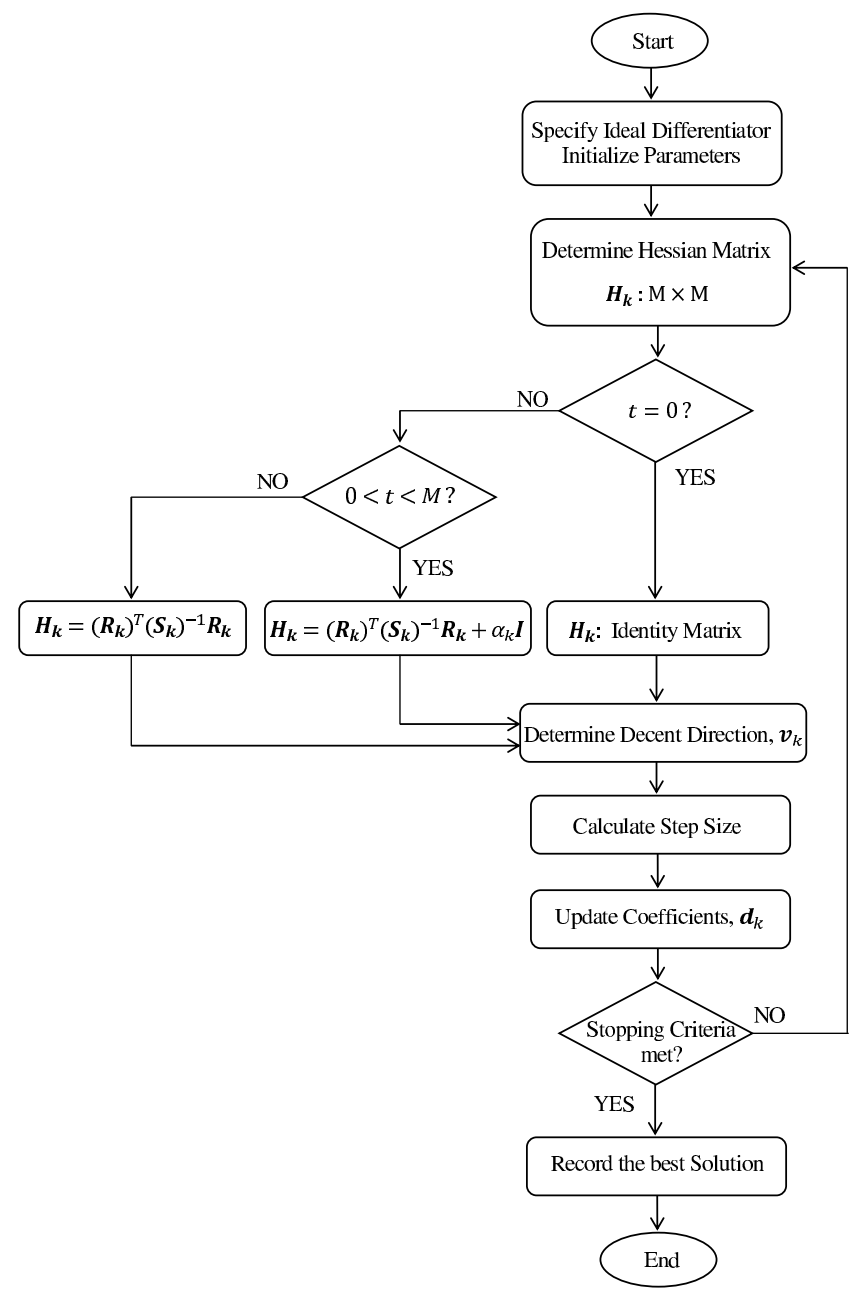

Fig. 1. Flowchart for the $L_{1}$ based FIR differentiator design method.

and window technique. The $L_{1}$ algorithm for the design of $(N-1)$ th order type-4 FIR differentiator with antisymmetric coefficients is described in this section.

The algorithm demands for the evaluation of first and second order derivative of the error function defined in (6). The $n$th component of gradient (first-order derivative) at $\mathbf{d}$ is given by

$$
\mathrm{g}^{n}(\mathbf{d})=\langle\cos (n \omega), \operatorname{sgn}(\varepsilon)\rangle
$$

where $\operatorname{sgn}(\varepsilon)$ gives the Signum result of the function $\varepsilon$.

The second order derivative of the error function, known as the Hessian matrix is computed over the entire digital frequency. It takes one of the three forms according to the number of zeros, $\left\{z_{1}, \ldots, z_{t}\right\}$ of $\varepsilon$ and is given by [22]

$$
\mathbf{H}(\mathbf{d})=\mathbf{R}^{T} \mathbf{S}^{-1} \mathbf{R}
$$

where $\mathbf{R}$ is a $t \times M$ matrix with $\mathbf{R}_{m n}=\sin \left(n z_{m}\right)$ and $z_{m}$ denotes the zero of the error function $\varepsilon$, at the $m$-th position. Also

$$
z_{m}=\frac{(2 m-1) \pi}{2 M}, \quad m=1,2, \ldots, M
$$

and $\mathbf{S}=\operatorname{diag}\left\{s_{1}, \ldots, s_{t}\right\}$ with $s_{m}=\frac{2 \mathbf{W}\left(z_{m}\right)}{\varepsilon^{\prime}}$. 
The modified Newton method generates a sequence of coefficients, $\mathbf{d}_{k}$, with number of iterations

$$
\mathbf{d}_{k+1}=\mathbf{d}_{k}-\alpha_{k}\left[\mathbf{H}_{k}\right]^{-1} \mathbf{g}_{k}
$$

and $\mathbf{v}_{k}$ is the Newton direction, given by

$$
\mathbf{v}_{k}=-\left[\mathbf{H}_{k}\right]^{-1} \mathbf{g}_{k} .
$$

It is assumed to be a descent direction. Here, $\mathbf{g}_{k}$ is the gradient of error function at $\mathbf{d}_{k}, \alpha_{k}$ is the step size and $\mathbf{H}_{k}$ is the Hessian matrix of $\varepsilon$ at the $k$ th iteration. In order to solve $\mathbf{v}_{k}$, the descent direction involves the solution of the linear equations with $M$ unknowns (the length of $\mathbf{v}_{k}$ ). To reduce these computations, the special structure of the matrix $\mathbf{H}_{k}$ in (8) is exploited based on the number of zeros of $\varepsilon$. The process flow chart is pictured in Fig. 1. The steps for the design of type-4 FIR differentiator based on $L_{1}$ criterion are summarized as:

Step 1: Design the ideal frequency response of type-4 digital differentiator, defined in (1). Set $M=N / 2$.

Step 2: Calculate initial vector $\mathbf{d}_{1} \in \mathfrak{R}^{M}$, set stopping condition factor, $\xi>0$, step-size selection parameters as $0<\sigma<1 / 2,0<\beta<1$ and for the control of Hessian matrix, set $\delta_{1}>0, \delta_{2}>0$ and $\mu>0$. Set $k=1$ to determine the coefficient, $\mathbf{d}_{1}$, using the relation

$$
\left(\begin{array}{ccc}
\cos \left(0 z_{1}\right) & \ldots & \cos \left(M z_{1}\right) \\
\cos \left(0 z_{2}\right) & \ldots & \cos \left(M z_{2}\right) \\
\vdots & \ldots & \vdots \\
\cos \left(0 z_{M+1}\right) & \ldots & \cos \left(M z_{M+1}\right)
\end{array}\right) \mathbf{d}_{1}=\left(\begin{array}{c}
H_{i d}\left(z_{1}\right) \\
H_{i d}\left(z_{2}\right) \\
\vdots \\
H_{i d}\left(z_{M+1}\right)
\end{array}\right)
$$

where $z_{m}$ is given by (9). This choice of initial solution vector is found to be optimal, verified by simulations.

Step 3: Determine the Hessian matrix $\mathbf{H}_{k}$ (second order derivative of error function) of size $M \times M$, based on the value of $t$, where $t$ represents the number of zeros of $\varepsilon$ and the zeros are considered to be simple [22].

(i) If $t=0$ or some zeros are not simple, then set Hessian matrix as identity matrix, $\mathbf{H}=\mathbf{I}$. Otherwise, compute the diagonal matrix, $\mathbf{S}_{k}$.

(ii) To ensure that the constructed matrix, $\mathbf{H}_{k}$ is positive definite, it is required the minimal distance between the zeros is beyond the threshold, $\mu$. Moreover, to guarantee global convergence, the elements of diagonal matrix, $\mathbf{S}_{k}$ is bounded by thresholds, $\delta_{1}$ and $\delta_{2}$. Thus, if $s_{\min }<\delta_{1}$ or $\delta_{2}<s_{\max }$, then set $\mathbf{H}=\mathbf{I}$. Otherwise, compute the matrix $\mathbf{R}_{k}$.

(iii) If $t \geq M, \delta_{1} \leq s_{\min }, s_{\max } \leq \delta_{2}$ and $\mu<\min _{p, q, p \neq q}\left|\cos \left(z_{p}\right)-\cos \left(z_{q}\right)\right|$, then set $\mathbf{H}_{k}=$ $\left(\mathbf{R}_{k}\right)^{T}\left(\mathbf{S}_{k}\right)^{-1} \mathbf{R}_{k}$, which is a positive definite matrix.

(iv) If $0<t<M, \delta_{1} \leq s_{\min }, s_{\max } \leq \delta_{2}$ or $\min _{p, q, p \neq q}\left|\cos \left(z_{p}\right)-\cos \left(z_{q}\right)\right| \leq \mu$, the matrix $\mathbf{H}_{k}$ is positive semidefinite. Set $\mathbf{H}_{k}=\left(\mathbf{R}_{k}\right)^{T}\left(\mathbf{S}_{k}\right)^{-1} \mathbf{R}_{k}+\alpha_{k} \mathbf{I}$, where $\alpha_{k}>0$.
Step 4: Determine the descent direction $\mathbf{v}_{k}$, a set of linear equations defined in (11), that obtains the unique solution and reduces the computational complexity by using the special structure of matrix $\mathbf{H}_{k}$.

Step 5: Algorithm stops if $\left|\left(\mathbf{v}_{k}\right)^{T} \mathbf{g}_{k}\right|$ is less than the given threshold, $\xi$.

Step 6: Calculate step-size, $\alpha_{k}$ according to the Armijo rule [22], to guarantee sufficient decrease of $\varepsilon$ at the $k$ th iteration.

Step 7: Update coefficients, set $\mathbf{d}_{k+1}=\mathbf{d}_{k}+\alpha_{k} \mathbf{v}_{k}$ and $k=k+1$. Go to Step 3 .

Step 8: The $M$ coefficients are stored and the frequency response of designed $(N-1)$ th order type-4 FIR differentiator is calculated.

\section{Design Examples}

The extensive experimental studies are carried out using MATLAB with different orders digital differentiators. The applicability of the proposed design algorithm is demonstrated by three examples, 5th, 7th and 9th order differentiators.

The $L_{1}$-method is formulated using the fact that the $L_{1}$-error function is certainly differentiable and this modified Newton method is developed for computing the differentiator coefficients [22]. The zeros of the differentiable $L_{1}$-error function are replaced with a different set of zeros at each iteration in order to decrease the error. The method is build with some set of constants called the control parameters. Chosen values of these parameters are: $\epsilon=10^{-6}, \sigma=10^{-3}, \beta=0.5$, $\delta_{1}=10^{-15}, \delta_{2}=10^{15}$ and $\mu=10^{-10}$. For simplicity, $W(\omega)$ is set to 1 . The coefficients of designed digital differentiator using the $L_{1}$-method is listed in Tab. 1 .

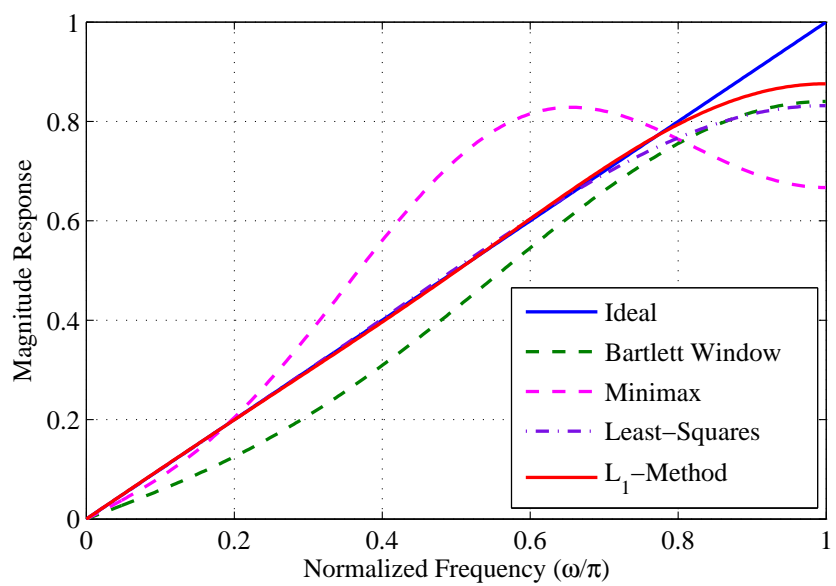

Fig. 2. Normalized magnitude response for 5 th order FIR digital differentiator. 


\begin{tabular}{|c|c|c|c|c|c|}
\hline \multirow{2}{*}{$\begin{array}{l}\text { Order } \\
\text { 5th }\end{array}$} & \multicolumn{5}{|c|}{ Optimized Coefficients } \\
\hline & $d(0)=-d(5)$ & $d(1)=-d(4)$ & $d(2)=-d(3)$ & & \\
\hline & 0.0209 & -0.1128 & 1.2411 & & \\
\hline \multirow[t]{2}{*}{ 7th } & $d(0)=-d(7)$ & $d(1)=-d(6)$ & $d(2)=-d(5)$ & $d(3)=-d(4)$ & \\
\hline & -0.0081 & 0.0341 & -0.1266 & 1.2620 & \\
\hline \multirow[t]{2}{*}{ 9th } & $d(0)=-d(9)$ & $d(1)=-d(8)$ & $d(2)=-d(7)$ & $d(3)=-d(6)$ & $d(4)=-d(5)$ \\
\hline & 0.0035 & -0.0140 & 0.0401 & -0.1321 & 1.2639 \\
\hline
\end{tabular}

Tab. 1. Optimized coefficients of FIR digital differentiator based on $L_{1}$-Method.

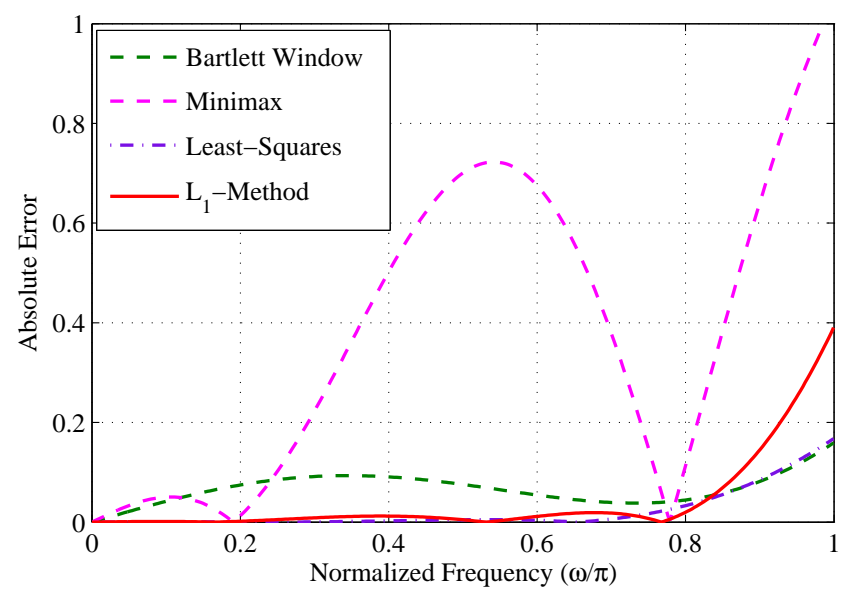

Fig. 3. Absolute magnitude error for 5th order FIR digital differentiator.

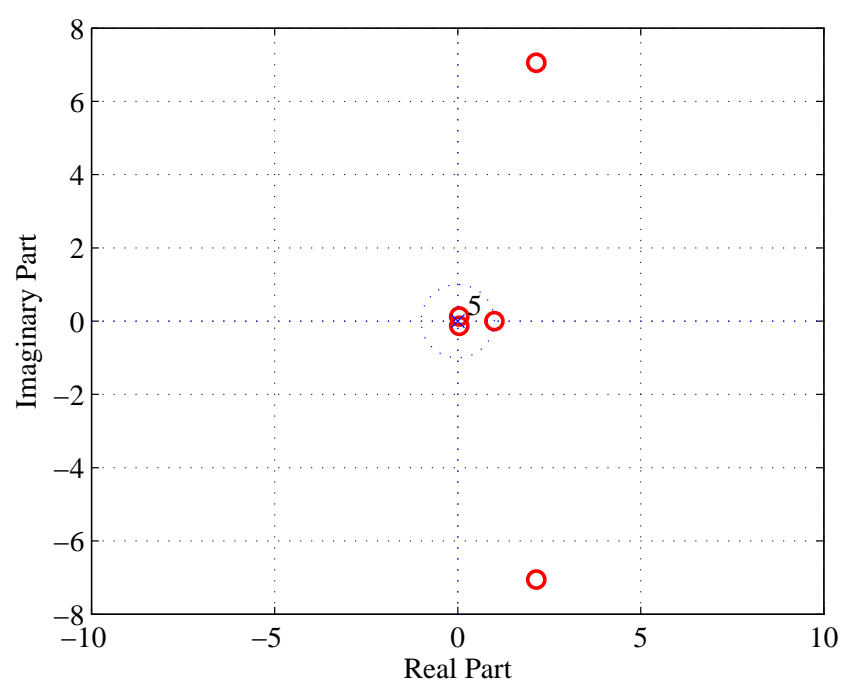

Fig. 4. Pole-zero plot for 5 th order FIR digital differentiator designed using $L_{1}$-method.

Figure 2 shows the comparison of magnitude response of the 5th order digital differentiator designed using the window technique, minimax method, least-squares design and the proposed $L_{1}$-method. It is remarked from Fig. 2, that the proposed differentiator based on $L_{1}$-method outperforms all other employed techniques. In order to study the effect of order on the design methods, similar plots are shown in Figures 5 and 8 for 7 th and 9th order, respectively.

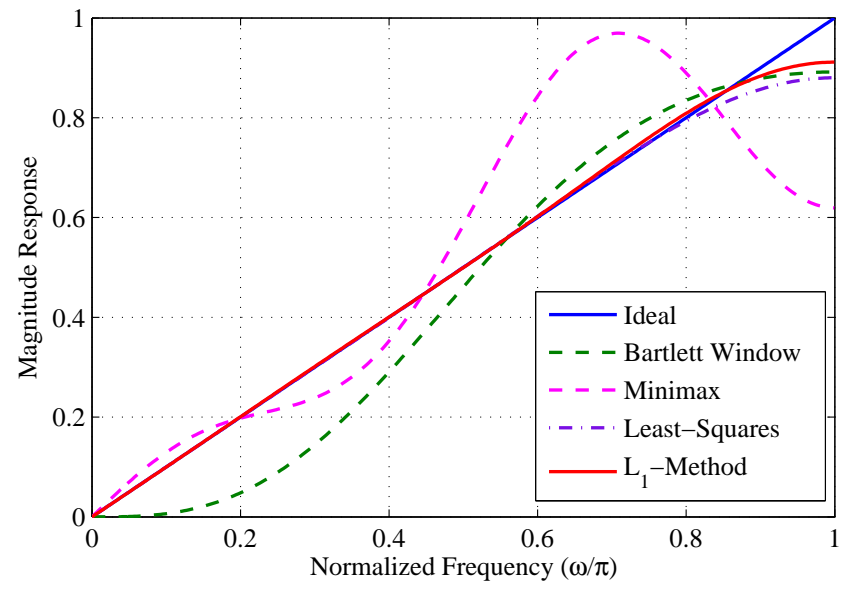

Fig. 5. Normalized magnitude response for 7 th order FIR digital differentiator

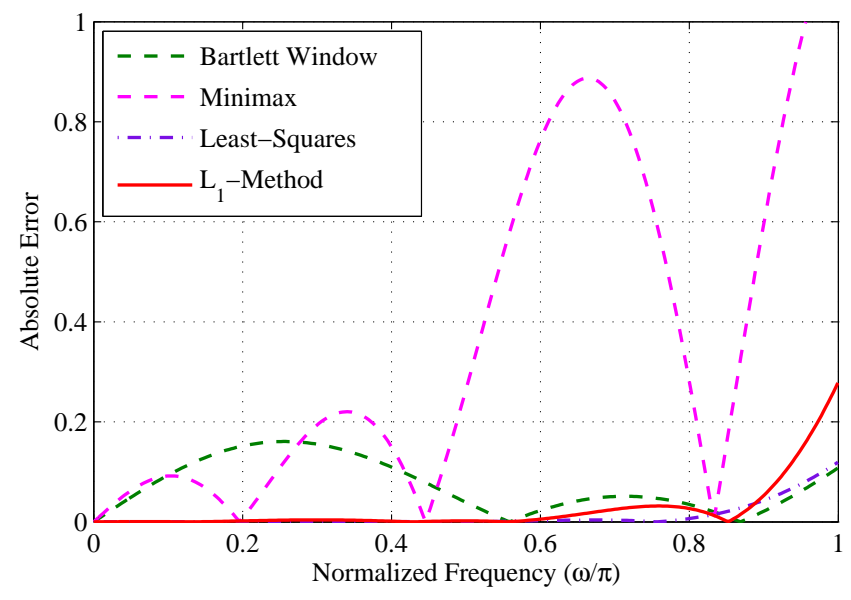

Fig. 6. Absolute magnitude error for 7 th order FIR digital differentiator.

It can be stated that with the increase in order, the response of the differentiator is improved at higher frequencies also.

The absolute magnitude error for the 5th, 7th and 9th order differentiator using all the reported methods is depicted in Figs. 3, 6 and 9, respectively. It can be seen from all the curves, that over a wide range of frequency, the proposed $L_{1}$-based differentiator exhibits least error. The absolute magnitude error for all design techniques are reported in Tab. 2, for the 5th, 7th and 9th order differentiators. Figures 4,7 and 10 demonstrates the pole-zero plot for the 5th, 7 th and 9th order digital FIR differentiators, respectively. 


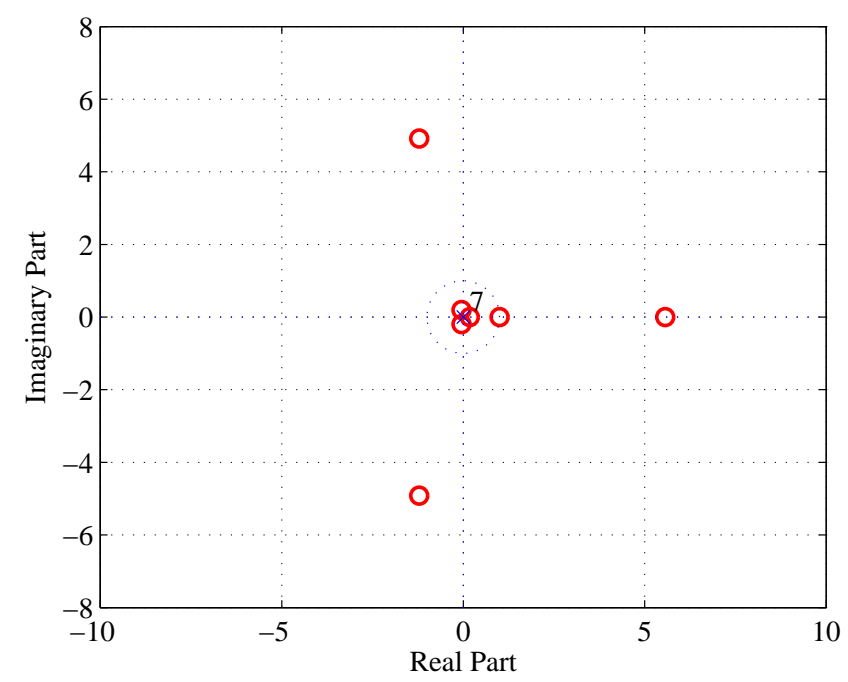

Fig. 7. Pole-zero plot for 7 th order FIR digital differentiator designed using $L_{1}$-method.

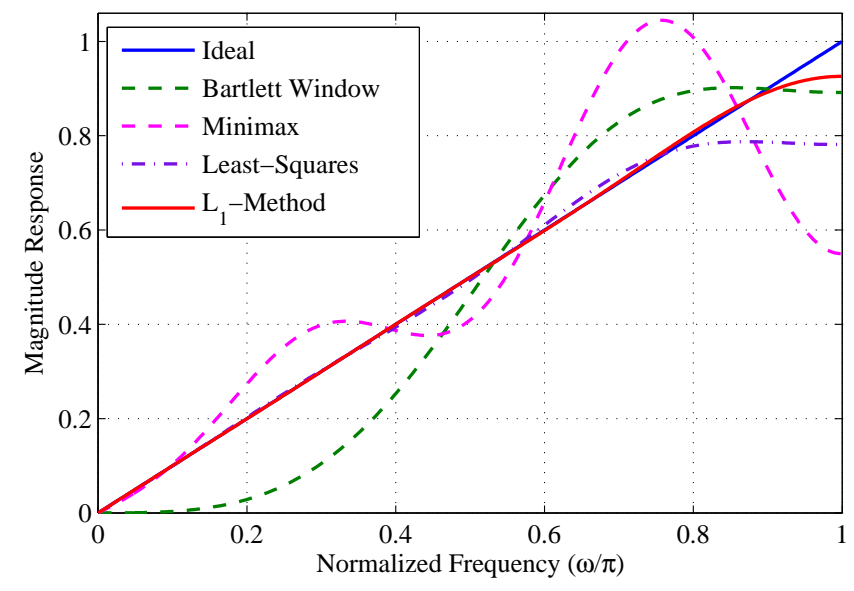

Fig. 8. Normalized magnitude response for 9 th order FIR digital differentiator

\begin{tabular}{llc}
\hline Order & Algorithm & Absolute Magnitude Error \\
\hline 5th & Bartlett Window & 2.1086 \\
& Minimax & 12.0177 \\
& Least-Squares & 1.3414 \\
& $L_{1}$-Method & 1.2408 \\
\hline 7th & Bartlett Window & 2.2835 \\
& Minimax & 11.7084 \\
& Least-Squares & 0.7557 \\
& $L_{1}$-Method & 0.7224 \\
\hline 9th & Bartlett Window & 3.1942 \\
& Minimax & 11.8469 \\
& Least-Squares & 0.8818 \\
& $L_{1}$-Method & 0.4917 \\
\hline
\end{tabular}

Tab. 2. Comparative analysis of absolute magnitude error of FIR digital differentiator.

The pole-zero plot guarantees the stability of the proposed differentiators with all poles inside the unit circle.

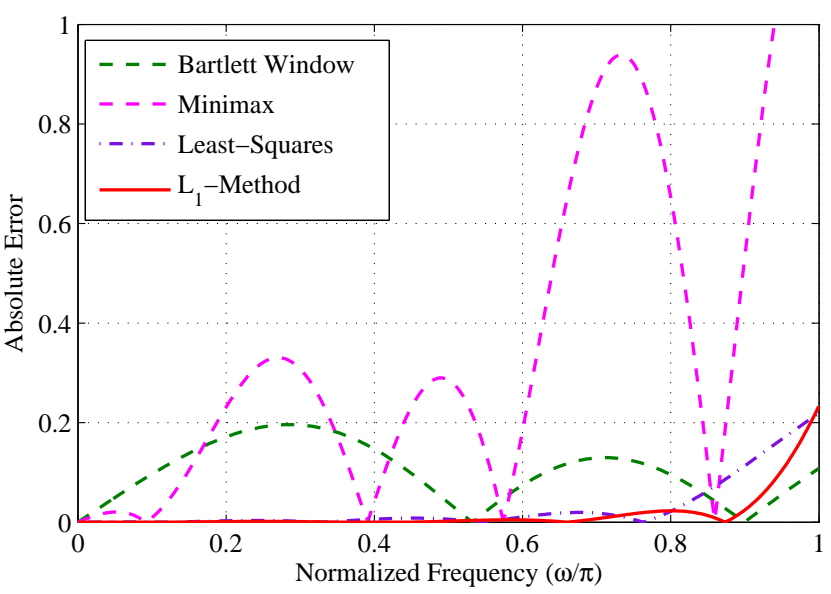

Fig. 9. Absolute magnitude error for 9th order FIR digital differentiator.

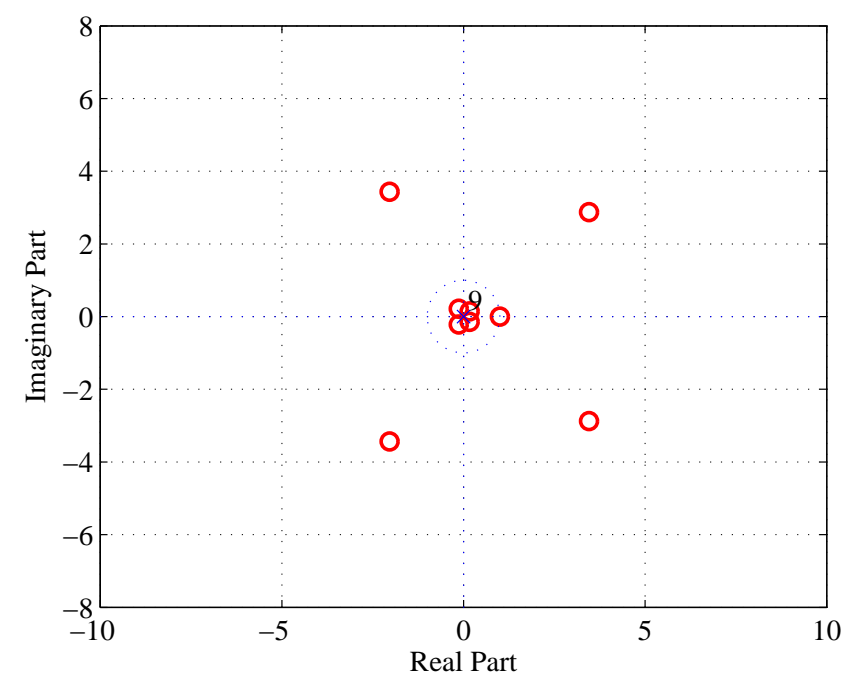

Fig. 10. Pole-zero plot for 9th order FIR digital differentiator designed using $L_{1}$-method.

Based on the observations, it is summarized that the proposed algorithm converges to the optimal solution for the digital differentiator design problem which depends on the initial values of the solution set. The designed FIR differentiator is stable as the location of all the poles are at origin. Furthermore, the properties of uniqueness, flattest response and differentiability of the fitness function, enhance the applicability of the proposed method for differentiator design problem.

\section{Conclusion}

This paper deals with the efficient design of FIR Digital differentiator using the $L_{1}$-method. The algorithm is based on the modified Newton method and is applied to compute the optimal coefficients on account of minimizing the absolute $L_{1}$-error. The results obtained clearly demonstrate the effective performance of the proposed method over the window technique, the minimax method and the least-squares approach design. It is concluded that, the $L_{1}$-based differentiator yield the flattest response over a wide frequency band 
with a unique solution and least absolute magnitude error. The proposed differentiator may be applied in the applications such as detection of edges in images, reading seismic data and prediction of earthquakes, detecting peaks in ECG signals, etc. As a future avenue of research, the design of half-band differentiator will be an interesting problem to be considered.

\section{References}

[1] SKOLNIK, M. I. Introduction to Radar Systems. New York (USA): McGraw- Hill, 1980. ISBN: 9780070579095

[2] XU, Y., DAI, T., SYCARA, K., et al. Service level differentiation in multi-robots control. In IEEE/RSJ International Conference on Intelligent Robots and Systems. Oct. 2010, p. 2224-2230. ISSN: 2153-0858. DOI: 10.1109/IROS.2010.5649366

[3] FUJIOKA, H. Characterizing approximated differentiators in digital control. In 11th International Conference on Control Automation Robotics and Vision. Dec. 2010, p. 923-926. DOI: 10.1109/ICARCV.2010.5707379

[4] MORGERA, S. D. Digital filtering and prediction for communications systems time synchronization. IEEE Journal of Oceanic Engineering, 1982, vol. 7, no. 3, p. 110-119. DOI: $10.1109 / J O E .1982 .1145524$

[5] LAGUNA, P., THAKOR, N. V., CAMINAL, P., et al. Low-pass differentiator for biological signals with known spectra: Application to ECG signal processing. IEEE Transactions on Biomedical Engineering, Apr. 1990, vol. 37, no. 4, p. 420-425. ISSN: 0018-9294. DOI: $10.1109 / 10.52350$

[6] AL-ALAOUI, M. A. Novel FIR approximations of IIR differentiators with applications to image edge detection. In 18th IEEE International Conference on Electronics, Circuits and Systems (ICECS). Dec. 2011, p. 554-558. DOI: 10.1109/ICECS.2011.6122335

[7] ZUBAIDAH, T., KANATA, B., RAMADHANI, C., et al. Comprehensive geomagnetic signal processing for sucessful earthquake prediction. In International Conference on Quality in Research (QiR). Jun. 2013, p. 212-219. DOI: 10.1109/QiR.2013.6632567

[8] KUMAR, B., ROY, S. C. D. Design of eficient FIR digital differentiators and Hilbert transformers for midband frequency ranges. International Journal of Circuit Theory and Applications, 1989, vol. 17, no. 4, p. 483-488. ISSN: 1097-007X. DOI: $10.1002 /$ cta.4490170409

[9] KUMAR, B., ROY, S. C. D. Maximally linear FIR digital differentiators for high frequencies. IEEE Transactions on Circuits and Systems, Jun. 1989, vol. 36, no. 6, p. 890-893. ISSN: 0098-4094. DOI: $10.1109 / 31.90411$

[10] ALAOUI, M. A. Novel digital integrator and differentiator. Electronics Letters, Feb. 1993, vol. 29, no. 4, p. 376-378. ISSN: 0013-5194. DOI: $10.1049 / \mathrm{el}: 19930253$

[11] BIHAN, J. L. Novel class of digital integrators and differentiators. Electronics Letters, May 1993, vol. 29, no. 11, p. 971-973. ISSN: 1053-587X. DOI: 10.1109/TSP.2008.929668

[12] UPADHYAY, D. K. Class of recursive wideband digital differentiators and integrators. Radioengineering, Sep. 2012, vol. 21, no. 3, p. 904-910. ISSN: $1805-9600$

[13] JAIN, M., GUPTA, M., JAIN, N. Linear phase second order recursive digital integrators and differentiators. Radioengineering, Jun. 2012, vol. 21 , no. 2 , p. 712-717. ISSN: 1805-9600
[14] NGO, N. Q. A new approach for the design of wideband digital integrator and differentiator. IEEE Transactions on Circuits and Systems II: Express Briefs, Sep. 2006, vol. 53, no. 9, p. 936-940. ISSN: 1549-7747. DOI: 10.1109/TCSII.2006.881806

[15] GUPTA, M., JAIN, M., KUMAR, B. Recursive wideband digital integrator and differentiator. International Journal of Circuit Theory and Applications, 2011, vol. 39, no. 7, p. 775-782. ISSN: 1097-007X. DOI: $10.1002 /$ cta.658

[16] UPADHYAY, D. K. Recursive wideband digital differentiators. Electronics Letters, Dec. 2010, vol. 46, no. 25, p. 1661-1662. ISSN: 00135194. DOI: $10.1049 / \mathrm{el} .2010 .2113$

[17] UPADHYAY, D. K., SINGH, R. K. Recursive wideband digital differentiators and integrators. Electronics Letters, 2011, vol. 47, no. 11, p. 647-648. ISSN: 0013-5194. DOI: 10.1049/el.2011.0420

[18] AL-ALAOUI, M. A. Class of digital integrators and differentiators. IET Signal Processing, 2011, vol. 5, no. 2, p. 251-260. ISSN: 1751-9675. DOI: 10.1049/iet-spr.2010.0107

[19] AL-ALAOUI, M. A., BAYDOUN, M. Novel wide band digital differentiators and integrators using different optimization techniques. In International Symposium on Signals, Circuits and Systems (ISSCS). Jul. 2013, p. 1-4. DOI: 10.1109/ISSCS.2013.6651225

[20] KUMAR, M., RAWAT, T. K. Optimal design of FIR fractional order differentiator using cuckoo search algorithm. Expert Systems with Applications, 2015, vol. 42, no. 7, p. 3433-3449. ISSN: 0957-4174. DOI: $10.1016 /$ j.eswa.2014.12.020

[21] GUPTA, M., RELAN, B., YADAV, R., et al. Wideband digital integrators and differentiators designed using particle swarm optimisation. IET Signal Processing, Aug. 2014, vol. 8, no. 6, p. 668-679. ISSN: 1751-9675. DOI: 10.1049/iet-spr.2013.0011

[22] GROSSMANN, L. D., ELDAR, Y. C. An $L_{1}$-method for the design of linear-phase FIR digital filters. IEEE Transactions on Signal Processing, Nov. 2007, vol. 55, no. 11, p. 5253-5266. ISSN: 1053-587X. DOI: 10.1109/TSP.2007.896088

[23] AGGARWAL, A., KUMAR, M., RAWAT, T. K. $L_{1}$ error criterion based optimal FIR filters. In Annual IEEE India Conference INDICON. Dec. 2014, p. 1-6. ISSN: 2325-940X. DOI: 10.1109/INDICON.2014.7030639

[24] AGGARWAL, A., RAWAT, T. K., KUMAR, M., et al. Intelligent Systems Technologies and Applications: Volume 1. 1st ed. Springer International Publishing, 2016. (An $L_{1}$-method: Application to digital symmetric type-II FIR filter design.) p. 335-343. ISBN: 978-3-319-23036-8. DOI: 10.1007/978-3-319-23036-8_29

[25] AGGARWAL, A., RAWAT, T. K., KUMAR, M., et al. Optimal design of FIR high pass filter based on $L_{1}$ error approximation using real coded genetic algorithm. International Journal Engineering Science and Technology, 2015, vol. 18, no. 4, p. 594-602. ISSN: 2215-0986. DOI: $10.1016 /$ j.jestch.2015.04.004

\section{About the Authors ...}

Apoorva AGGARWAL received her B.Tech in Electrical \& Electronics Engineering in the year 2011 from GGSIPU, Delhi. She received her M.Tech, exemplary performance, from GGSIPU, Delhi in 2013. She is currently pursuing $\mathrm{Ph} . \mathrm{D}$. in the area of optimal design of digital filters from Netaji Subhas Institute of Technology, University of Delhi.

Tarun Kumar RAWAT received his M.Tech and Ph.D. from NSIT, University of Delhi in the year 2003 and 2010, respectively. Presently, he is associated with NSIT, Delhi, India, as Assistant Professor in the Department of Electronics and 
Communication Engineering. His research interests include VLSI signal processing, digital signal processing, statistical signal processing and filter optimization via nature-inspired computational techniques. He has published more than 30 research papers in international journals and two books: Signal and System, Digital Signal Processing in Oxford University Press.

Manjeet KUMAR received his B.Tech in Electronics \& Communication Engineering in the year 2008 from Kurukshetra University, Haryana. He received his M.Tech from Ambedkar Instiute of Technology, GGSIPU, Delhi in 2011. He is currently pursuing Ph.D. in the area of optimal design of fractional order digital filter from Netaji Subhas Institute of Technology, University of Delhi.

Dharmendra Kumar UPADHYAY received his $\mathrm{Ph}$.D. from Uttrakhand Technical University, Uttrakhand in the year 2013. Presently, he is associated with Netaji Subhas Institute of Technology, Delhi, India, as Associate Professor in the Department of Electronics and Communication Engineering. His research interests include digital signal processing, microwave and array antenna design and filter optimization via nature-inspired computational techniques. He has published more than 20 research papers in international journals. 